\title{
ZEOLITE MEMBRANES FROM KAOLIN
}

B. G. KARLE* , C. J. BRINKER ${ }^{*}, \dagger$ and M. L. F. PHILLIPS $\dagger$ CONF- $960401--6 /$

* Center for Micro-Engineered Ceramics, University of New Mexico, Albuquerque, NM 87131.

$\uparrow$ Sandia National Laboratories, Albuquerque, NM, 87185

\section{ABSTRACT}

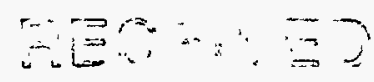

Zeolite films are sought as components of molecular sieve membranes. Different routes thed to prepare zeolite composite membranes include growing zeolite layers from gels on porous supports, depositing oriented zeolites on supports, and dispersing zeolites in polymeric membranes. In most cases, it is very difficult to control and avoid the formation of cracks and/or pinholes. Our approach to membrane synthesis is based on hydrothermally converting films of layered aluminosilicates into zeolite films. We have demonstrated this concept by preparing zeolite A membranes on alumina supports from kaolin films. We have optimized the process parameters not only for desired bulk properties, but also for preparing thin (ca. $5 \mu \mathrm{m}$ ), continuous zeolite A films. Scanning electron microscopy shows highly intergrown zeolite A crystals over most of the surface area of the membrane, but gas permeation experiments indicate existence of mesoporous defects and/or intercrystalline gaps. It has been demonstrated that the thickness of the final zeolite A membrane can be controlled by limiting the amount of precursor kaolin present in the membrane.

\section{INTRODUCTION}

Light gas separation is a major topic of membrane research. This is because membranes can be used for such energy intensive processes as isolation of air products, purification of natural gas, and separation of product streams in fuel refining. Gas membranes that are now used commercially are of two types: inorganic microporous membranes that separate gases based on differences in molecular weights (Knudsen diffusion) or vapor pressure (pervaporation), and polymer membranes that separate gases based on differences in their solubilities and diffusivities in the polymer matrix [1].

In contrast, a molecular sieve membrane would reject gases whose kinetic diameters exceed the pore dimensions of the membrane. This technology promises increased separation factors relative to existing gas membranes. Microporous solids such as zeolites are an obvious choice for the discriminating elements in such membranes, and are in fact currently used as molecular sieves to separate atmospheric gases in the pressure-swing adsorption (PSA) process [2]. Pore size can be modified by selecting phase, and can be tunable within a given phase by ion exchange.

The economics of gas separation on a large scale may not favor zeolite membranes over polymer membranes, PSA, or cryogenic separation in all applications. However, we might expect inorganic membranes to compete well in high temperature separations (ca. $500-600^{\circ} \mathrm{C}$ ), such as removal from $\mathrm{H}_{2}$ from alkanes in petrochemical refining, and from $\mathrm{NH}_{3}$ in the Haber process.

While zeolites are generally stable at such temperatures, differences in expansion of the film and substrate can cause the membrane to crack or peel during thermal cycling. Other issues critical to the development of zeolite membranes include thickness and leakage. Films should be as thin as 


\section{DISCLAIMER}

Portions of this document may be illegible in electronic image products. Images are produced from the best available original document. 


\section{DISCLAIMER}

This report was prepared as an account of work sponsored by an agency of the United States Government. Neither the United States Government nor any agency thereof, nor any of their employees, makes any warranty, express or implied, or assumes any legal liability or responsibility for the accuracy, completeness, or usefulness of any information, apparatus, product, or process disclosed, or represents that its use would not infringe privately owned rights. Reference herein to any specific commercial product, process, or service by trade name, trademark, manufacturer, or otherwise does not necessarily constitute or imply its endorsement, recommendation, or favoring by the United States Government or any agency thereof. The views and opinions of authors expressed herein do not necessarily state or reflect those of the United States Government or any agency thereof. 
possible to maximize permeability, but must also simultaneously minimize leakage through intercrystalline gaps, cracks, or pinholes.

Pure zeolitic crystalline films are very brittle and need careful handling [3]. Such films are generally deposited upon porous supports that are more permeable than the zeolite films themselves. In such a composite membrane, the support does not contribute to the separation. The zeolite layer acts as the selective layer, but also limits the permeability of the membrane. Traditionally, zeolite membranes have been synthesized by precipitating polycrystalline films of the desired zeolite phase onto porous supports [4-8]. Even though continuous intergrown films have been reported, it is very difficult to totally eliminate pinholes and defects from membranes made by such a method. Films have been "caulked" with organic binders in order to inhibit leakage [9]. Caveats in this approach include permeation of gas through the binder phase, obstruction of the zeolite pores, and degradation of the binder at elevated temperatures $\left(>150^{\circ} \mathrm{C}\right)$.

An alternate approach is to prepare a film from zeolite precursors, then convert this precursor film into zeolite film by means of an appropriate synthesis technique [10]. The advantage of this method is that defects and pinholes might be eliminated even before the zeolite crystals take shape. In our approach, we start with a precursor that may minimize the possibility of pinholes by virtue of its structure. One such precursor is the clay mineral kaolin, an aluminosilicate with the empirical formula $2 \mathrm{SiO}_{2} \cdot \mathrm{Al}_{2} \mathrm{O}_{3} \cdot 2 \mathrm{H}_{2} \mathrm{O}$. Upon heat treatment, kaolin is converted to dehydrated, amorphous metakaolin. This phase of course retains the original Si/Al stoichiometry of 1:1, which is also the $\mathrm{Si} / \mathrm{Al}$ ratio in the zeolite Linde Type A (LTA). LTA has a cubic structure with three dimensional channels, with pore diameters that can be tuned from approximately 3.0 to $4.5 \AA$ through ion exchange [2]. This pore size range makes this phase a very good candidate for separations such as natural gas purification, e.g., separating $\mathrm{CH}_{4}(3.8 \AA)$ from $\mathrm{CO}_{2}(3.3 \AA), \mathrm{H}_{2} \mathrm{~S}$ (3.6 $\AA)$, and $\mathrm{H}_{2} \mathrm{O}(2.65 \AA)$.

Because it is amorphous, metakaolin is readily converted into sodium LTA (NaA) by hydrothermal treatment with sodium hydroxide $[1,11]$. In addition, the plate-like shape of original kaolin particles is retained by metakaolin particles. These platelets can be used to prepare a highly continuous clay film on top of a porous support by means of a simple slip casting process [12]. Thus, the specific structure and reactivity of metakaolin films make them excellent candidates as precursors of zeolite membranes. It is also conceivable that the morphology of the clay particles may drive the LTA to crystallize in a dense, semi-ordered mosaic, and that residual nutrient may act to prevent leakage between the crystallite facets.

In this work, we report the experimental conditions used to prepare zeolite membranes from kaolin, and describe how process parameters affect the physical characteristics of the membranes. In addition, gas permeation results are presented, and discussed in the context of membrane structure.

\section{EXPERIMENTAL}

\section{SYNTHESIS}

The process of transforming kaolin into metakaolin, then into zeolite A involves several steps. First, kaolin (Aldrich) was heated at $800^{\circ} \mathrm{C}$ for one hour to convert it to amorphous metakaolin. The metakaolin was then contacted with the appropriate quantity of aqueous sodium hydroxide 
solution (Baker) to maintain the reactant composition $5 \mathrm{Na}_{2} \mathrm{O} \cdot \mathrm{Al}_{2} \mathrm{O}_{3} \cdot 2 \mathrm{SiO}_{2} \cdot 200 \mathrm{H}_{2} \mathrm{O}$. This mixture was kept in a closed Teflon vessel. After aging at room temperature for $24-48$ hours, this vessel was transferred to an oven maintained at $100^{\circ} \mathrm{C}$ for $1-4$ hours. After this crystallization period the solid zeolite crystals were separated from the liquid phase, washed with deionized water, and filtered. This process yielded bulk zeolite A powder. This material was then calcined and characterized for particle size and purity.

\section{FILM PREPARATION}

Kaolin films were deposited onto the surfaces of porous alumina support discs. These supports were prepared by cold-pressing corundum with particle sizes from 0.5 to $3 \mu \mathrm{m}$, and hence contain similarly sized pores [13]. The support discs were polished to a surface roughness of $15 \mu \mathrm{m}$. With a typical particle size of kaolin particles of less than $1 \mu \mathrm{m}$, it was very easy to obtain a good penetration and coverage of the entire surface of the flat support by kaolin platelet layers. An optimum thickness of such a kaolin film was found to be between 5 and $10 \mu \mathrm{m}$.

There are a variety of methods that can be used for preparation of these kaolin films. In most cases, we began with a highly dilute $(<0.1 \mathrm{~g} / \mathrm{L})$, stable suspension of kaolin in water. It is essential for this suspension to be free of impurities and dust particles. Sonication, deflocculants and continuous stirring are used to ensure that the kaolin particles do not form aggregates in the suspension prior to deposition. This suspension was then either vacuum filtered, centrifuged, or slip cast onto the top surface of the clean, polished alumina disc. After drying overnight, the films are then calcined very slowly $\left(2{ }^{\circ} \mathrm{C} / \mathrm{min}\right)$ to $800^{\circ} \mathrm{C}$ in order to convert the kaolin into metakaolin. Requisite amount of $\mathrm{NaOH}$ (according to the stoichiometry mentioned above) was then added to this film in a closed reaction vessel. After the room temperature aging step, the vessel was transferred to a preheated oven for crystallization. After the reaction was complete, the films were washed carefully to remove all alkali residue and dried overnight prior to characterization.

\section{CHARACTERIZATION}

Powder X-ray diffraction was used to identify the phases in the raw materials and the reaction products. All diffraction patterns were obtained on a Scintag $\theta-2 \theta$ Diffractometer using $\mathrm{CuK}_{\alpha}$ radiation $(\lambda=1.54059 \AA)$ with a scan rate of $1 \%$ min and a step size of $0.02^{\circ}$. An Environmental Scanning Electron Microscope (ElectroScan E3) was used to examine the morphology of crystals and films obtained. The films were tested for membrane performance by sealing the edges of the substrate, then measuring the permeability of different gases through the films using an automated gas permeation system. The flow rates of individual gases were measured as functions of pressure drop across the membrane and the temperature of the system.

\section{RESULTS}

Nucleation of LTA crystals takes place during aging, wherein the template $(\mathrm{NaOH})$ reacts with the metakaolin nutrient at room temperature. The longer this aging time, the more nuclei available for growth of crystals. If aging is continued for extended periods of time (longer than 10 days), hydrothermal treatment yields small $(<0.6 \mu \mathrm{m}) \mathrm{NaA}$ crystals. For the purposes of our experiments we limited this aging process to a period of approximately two days.

The process of crystallization is much faster at elevated temperatures. The temperature chosen for this next step can determine not only the crystalline phase that precipitates, but also the size of 
those crystals. Pure zeolite A crystals precipitated at approximately $100^{\circ} \mathrm{C}$. At this temperature, crystals in the size range of $1-2 \mu \mathrm{m}$ were obtained.

The total yield of crystalline zeolite increases with reaction time. The SEM pictures in Figure 1 follow the progress of LTA crystallization with time at $100{ }^{\circ} \mathrm{C}$ after an aging period of approximately 48 hours. Figure 2 follows the same progress with X-ray diffraction patterns of samples at different time intervals. As is visible in these figures, crystals slowly began nucleating and precipitating following a period of incubation (no crystallization). The change of structure takes place after about an hour after the reaction begins; this is evident from the tiny spheres in the SEM image and small peaks of zeolite A crystals appearing in the XRD pattern.

Understanding the bulk process of conversion of metakaolin into $\mathrm{NaA}$ led us to translate that process to the preparation of $\mathrm{NaA}$ films. The reaction parameters were maintained at the same values as that used for the reaction above, and applied to a film of kaolin prepared on porous alumina disc supports. The most significant aspect of this process was that it was very easy to control the amount of clay being deposited, and hence we could readily dictate the thickness of our membrane.

We found that the thickness of the clay film is key to obtaining crack-free zeolite films. Figure 3 shows the top surface of one such LTA film where the thickness of the film caused it to crack at several places during the formation process. This film was approximately $100 \mu \mathrm{m}$ thick. We subsequently reduced the amount of clay for available for deposition to yield a $5 \mu \mathrm{m}$ thick film. Conversion of such thin kaolin films ultimately led to preparation of crack-free zeolite films. An example of one such films is depicted in Figure 4, which shows the top surface of a highly intergrown crystalline LTA film. Interestingly, the entire surface of the membrane was observed to have a similar morphology. With an average particle size of ca. $1 \mu \mathrm{m}$, this film was approximately 5-7 $\mu \mathrm{m}$ thick when observed in cross section. The process of bulk synthesis of LTA from metakaolin was thus successfully translated to preparing thin, continuous zeolite A films.

Once the NaA films were prepared, they were subjected to permeation measurements to determine whether they functioned as molecular sieve membranes. Figure 5 is a plot of permeance of several gases of interest as a function of average pressure across the membrane. Uncoated alumina supports yielded hardly any separation of permanent gases, with permeance values of approximately $10^{-2}\left[\mathrm{cc}(\mathrm{STP}) \cdot \mathrm{cm}^{-2} \bullet \mathrm{s}^{-1} \bullet \mathrm{cm} \mathrm{Hg}^{-1}\right]$. Permeation behavior of a representative LTA membrane is very similar to that of a bare support, except that the permeance is reduced by an order of magnitude. The dependence of permeance on the average pressure indicates that the flow through the pores is occurring mainly through viscous and Knudsen flow. In the viscous flow regime, permeance is inversely proportional to the viscosity of the gas. In the Knudsen flow regime, the permeance is governed by the gas molecules' mean free path, which makes permeance inversely proportional to the square root of the molecular weight of the gas. For example, for helium and nitrogen, viscous flow at room temperature dictates a separation factor of about 1.2 , while Knudsen flow dictates a separation factor of about 2.65 .

A closer look at the numbers on the plot in Figure 5 indicates that the selectivity of helium over nitrogen is somewhere in the range of 2.0 . This implies that trans-membrane flow is occurring by a combination of viscous and Knudsen mechanisms. This in turn suggests the presence of nonselective pathways alternative to the microporous channels in LTA crystals. These may be a combination of mesoporous (10-100 $\AA$ ) and macroporous (100-1000 $\AA$ ) flaws; the former being 
intercrystalline gaps, the latter pinholes not detected in SEM. We are currently exploring methods for ripening the LTA crystals to merge the pinholes, and for caulking the intercrystalline gaps with inorganic polymers.

\section{CONCLUSIONS}

We have demonstrated that a Linde Type A zeolite composite membrane can be synthesized directly from a kaolin film deposited on a porous support. The parameters affecting the conversion of kaolin into zeolite A have been optimized for preparing continuous polycrystalline LTA films 5-10 $\mu \mathrm{m}$ thick. The thickness of the zeolite film is controlled very effectively by the quantity of kaolin precursor available; thinner films $(<10 \mu \mathrm{m})$ are less prone to cracking. The hoped-for benefit of reduction of intercrystalline gas leakage due to the presence of residual amorphous precursor has not yet materialized. Post-synthesis treatments such as caulking and ripening the zeolite grains with extra nutrient are being evaluated.

\section{ACKNOWLEDGMENTS}

This work was funded by the U. S. Department of Energy under contract \#DE-AC04-94AL85000.

\section{REFERENCES}

1. Koros, W. J. "Gas Separation" in Membrane Separation Systems, R.W. Baker et al., Eds.; Noyes Data Corp.: Park Ridge, NJ, 1991, pp. 189-241.

2. Breck, D. W. Zeolite Molecular Sieves: Structure Chemistry and Use, John Wiley and Sons: New York, 1974.

3. Tsikoyiannis, J. G.; Haag, W. O. Zeolites, 1992, 12, 126-30.

4. Geus, E. R.; Mulder, A.; Vischjager, D. J.; Schoonman, J, van Bekkum, H. Key Engineering Materials, 1991, $61 \& 62,57-64$.

5. Geus, E. R.; den Exter, M. J.; van Bekkum, H. J. Chem. Soc. Farad. Trans. I 1992, 88, 3101-09.

6. Geus, E. R.; van Bekkum, H.; Bakker, W. J. W.; Moulijn, J. A. Microporous Materials, 1993, 1, 131-47.

7. Jia, M-D.; Peinemann, R-V.; Behling, R-D. J. Membr. Sci. 1993, 82, 15-26.

8. Yan, Y.; Davis, M. E.; Gavalas, G. R. Ind. Eng. Chem. Res. 1995, 34, 1652-61.

9. Te Hennepe, H. J. C.; Boswerger, W. B. F.; Smolders, C. A.; Borgeman, D.; Mulder, M. H. V. J. Membr. Sci. 1994, 89, 185-96.

10. Ma, Y. H.; Xiang, S. U.S. Patent 5,258,339.

11. Rees, L.V.C.; Chandrasekhar, S. Zeolites, 1993, 13, 524-533.

12. Nadeau, P. H. Applied Clay Science 1987, 2, 83-93.

13. These supports were also obtained from Golden Technologies Corp., Golden, CO. 

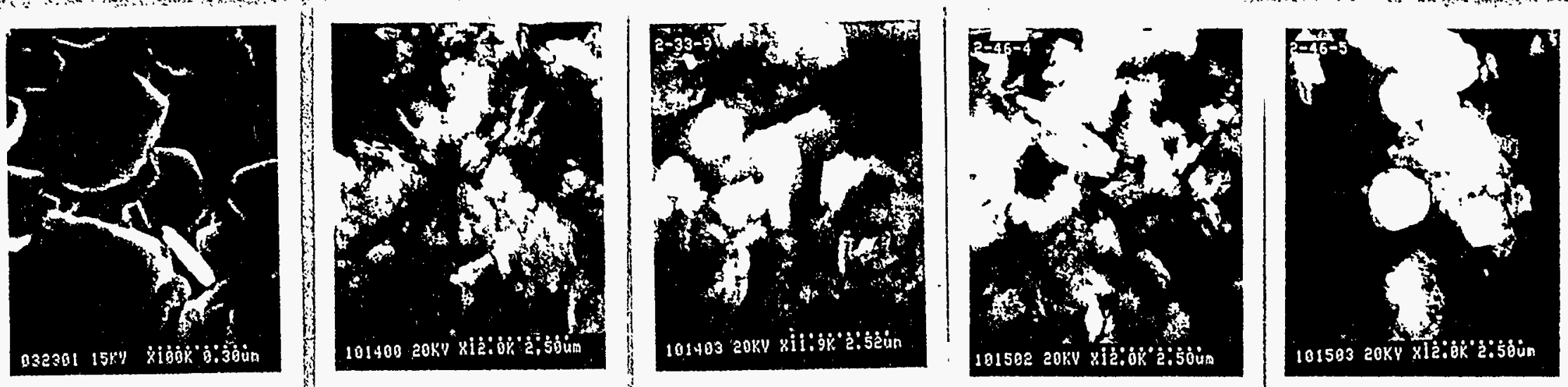

Metakaolin Particles

Figure 1. Evolution of Zeolite A Crystals over Time:

$$
\text { (1) }
$$

The reaction was carried out at $100^{\circ} \mathrm{C}$ after a 48 hour period of aging at room temperature. Samples were taken out at every 5 minutes upto 4 hours. This figure shows representative images

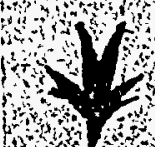
Zeolite A Crystals of samples taken out during this process. They are arranged clockwise from top-left to bottom-left
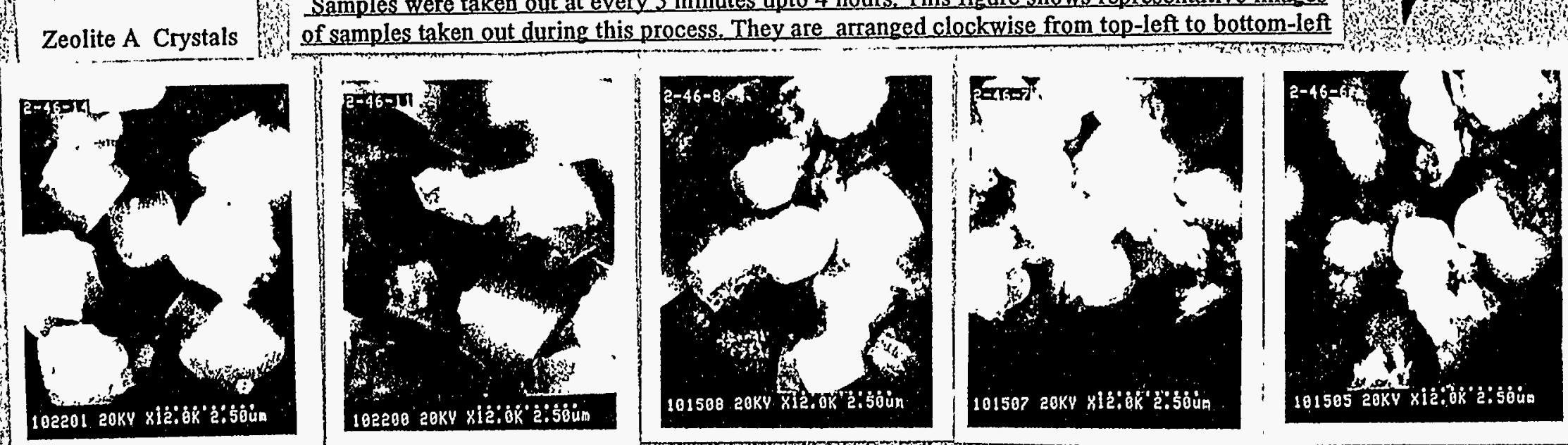

गएकाल

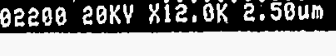

15. 


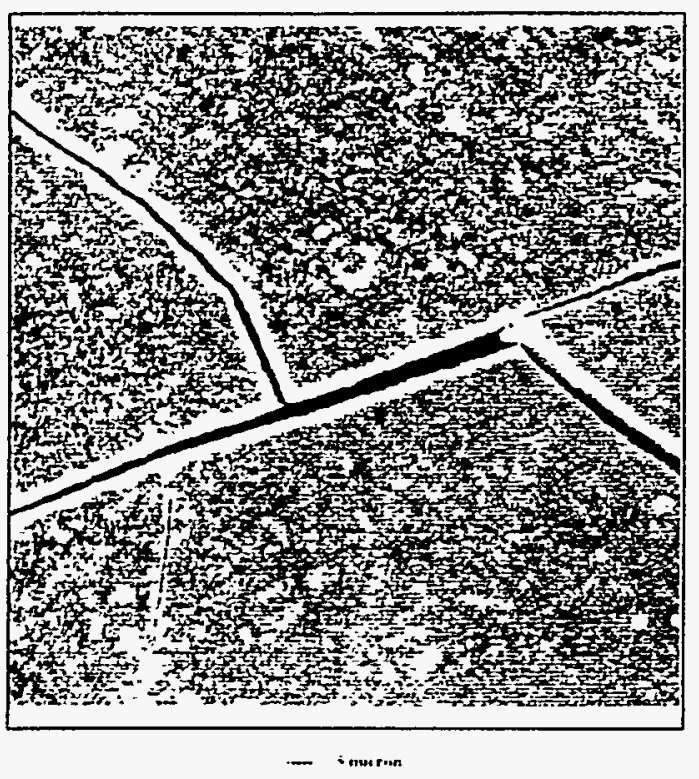

Figure 3. Cracked Zeolite A Film ( 100 um thicis)

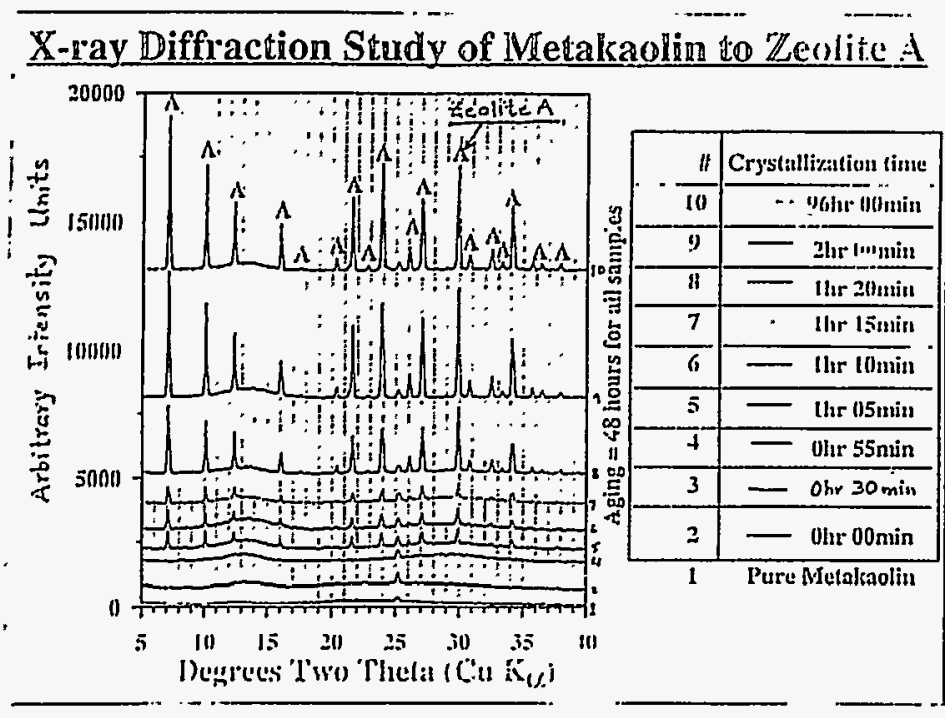

Figure 2. XRD Analysis of Evolution of Zeolite A from Melakaolin

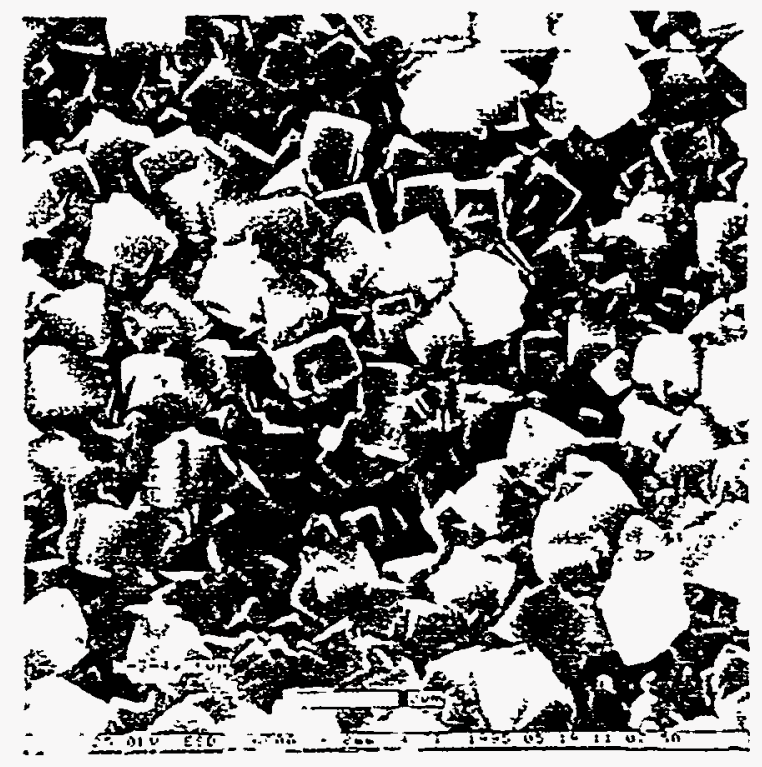

Figure 4. Intergrown Crystals in a Zeolite A Film Made from $\mathrm{NaOH}$ and Metakaolin

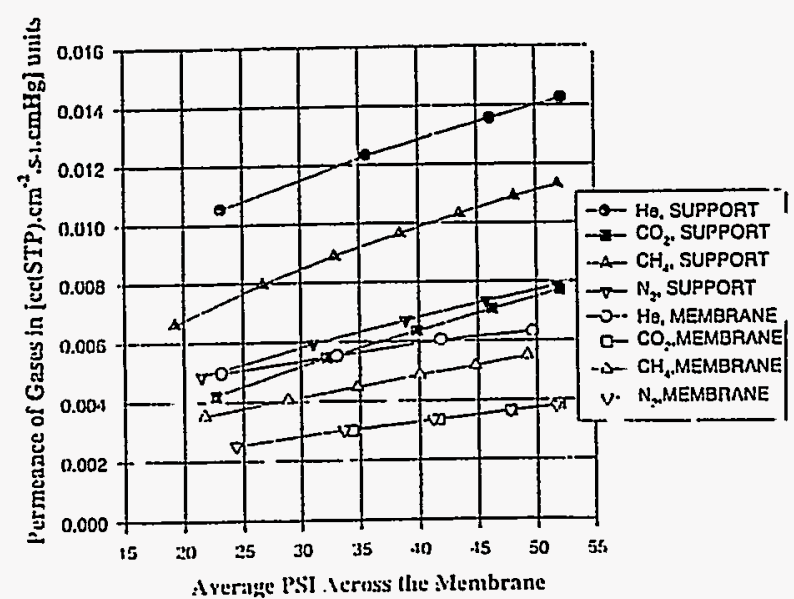

Figure 5. Permeation Behavior of a Typical Zeolite A Membrane on $\alpha$-Alumina Support Indicates a combination of Knudsen and Viscous Flows with No Molecular Sieving 